\section{Partizipation in Politik und Wissenschaft und ihre Schwierigkeiten} Bericht zur Tagung „Partizipationsfor-
schung und Partizipationsverfahren in der
sozialwissenschaftlichen Klimaforschung“

Müncheberg, 25.-26. April 2012

\section{von Martin Sand, ITAS}

Partizipation und Klimaforschung - wie passt das zusammen? Ein vornehmlich junges Kolloquium von überwiegend Sozial- und Politikwissenschaftlern widmete sich an beiden Workshoptagen einem Komplex aus Fragen zur Partizipation. Dabei wurden in Vortragsblöcken Beispiele aus der Klima- und Klimafolgenforschung vorgestellt sowie theoretische Aspekte der Partizipationsforschung präsentiert. Ausgedehnte Diskussionsrunden und eher unkonventionelle Verfahren (,Speed Poster Dating“) regten zum kreativen Austausch an. Die Tagung selbst wurde durch ihre anregenden Methoden zu einem Vorzeigebeispiel gelungener Organisation.

\section{Allgemeiner Erwartungshorizont und Kontext}

Von der Klima- und der Klimafolgenforschung, in der schon seit geraumer Zeit regionale Akteure in Forschungsprozessen eingebunden werden, erhofften sich die Teilnehmer einen ersten Einblick in unterschiedliche Partizipationspraktiken. In diesem stark anwendungsorientierten Feld mit unmittelbaren Handlungsfolgen konnte man eine von den betroffenen Akteuren und Forschern beiderseits motivierte Transdisziplinarität mit nunmehr langjährigen Erfahrungen kennenlernen. Interessant war zu beobachten, wie sich in den Diskussionen häufig der Versuch abzeichnete, den Partizipationsbegriff aus politischen Kontexten auf die wissenschaftliche Partizipation zu übertragen und einen Begriff für erfolgreiche Partizipation, der auf beide Kontexte, Wissenschaft und Politik, anwendbar ist, zu entwickeln - ein allzu ambitioniertes Unterfangen. Es stellte sich heraus, dass es wesentlich einfacher ist, einen negativen Begriff von erfolgreicher Partizipation aufzufin- den also anzugeben, was explizit nicht darunter zu verstehen sei. Die im Folgenden vorgestellten Beiträge widmeten sich verstärkt den Partizipationsprozessen. Die Klimaforschung selbst stand bei nur wenigen Vorträgen der Tagung im Fokus.

\section{Theoretische Aspekte der Partizipation}

Im Keynote-Vortrag unternahm Heike Walk (Zentrum Technik und Gesellschaft, TU Berlin) den Versuch, eine Taxonomie möglicher allgemeiner Motivationen für Partizipation herauszuarbeiten. Walk unterschied dabei drei Grundströmungen: die demokratische, die ökonomische und die emanzipatorische Funktion. Die erste Motivation diene der Erhöhung von Akzeptanz und Legitimität von Entscheidungen und lehne sich dabei an ein Verständnis einer bürgernahen Demokratie an. Bei der zweiten Motivlage erhofften sich die Akteure durch eine verbesserte Kommunikation und das Verwerten verschiedener Wissensbestände und Perspektiven effizientere Resultate, so Walk. Die emanzipatorische Funktion stehe in enger Beziehung zur demokratischen. Mit dieser dritten Motivlage gehe die Hoffnung auf ein steigendes Verantwortungsbewusstsein der beteiligten Akteure und die Ausprägung nachhaltiger Lernprozesse einher. Es ist klar, dass solche Modellierungsversuche, wie Walk hier einen unternommen hat, die Wirklichkeit immer in gewisser Weise „einengen“.

Damit ist dieses Unterfangen jedoch nicht sinnlos. Vielmehr gilt es, die Begriffe weiter zu präzisieren und die Unterschiede zu anderen Begriffen schärfer zu konturieren. Folgerichtig stellten sich die Tagungsteilnehmer in der anschließenden Diskussion den Fragen, wo spezifische Beispiele einzuordnen seien, welche Motivationen nicht berücksichtigt wurden und wie diese Motivationen klarer beschrieben werden könnten. In der Realität dürfte eine Gemengelage der drei genannten Motivationen, die jeweils unterschiedlich stark gewichtet werden, die Akteure zur Partizipation bewegen. Man würde erwarten, dass jedenfalls in der Klimaforschung die Motivation vonseiten der Politik in der Steigerung der Akzeptanz der darauf folgenden Klimaanpassungsmaßnahmen liege. Eine breitere empirische Analyse, die die Zusammenhänge zwischen den 
Charakteristika und den Motivationen der Akteure herausarbeitet, steht hier noch aus.

Dass teilweise auch dort, wo öffentlich mit dem Begriff Partizipation geworben wird, Missstände im Verständnis und in der Umsetzung von Partizipation offen zu Tage treten, zeigte Cordula Kropp (Fakultät für angewandte Sozialwissenschaften, Hochschule München) in ihrem Vortrag über die ,Strukturprobleme des expertokratischen Staats". Sie beschrieb detailliert eine vom BMBF lancierte Veranstaltung zur Energiewende in München, die sich als Expertenforum mit informeller Partizipation herausstellte. Die Architektur dieser Veranstaltung die Experten stehend auf der Bühne, das Publikum im unbeleuchteten Saal unten unterstrichen die hierarchische Asymmetrie von Wissenschaft und Laientum. Diese Konstellation würde Partizipation oder einen jeden Dialog bereits ex ante unterbinden, so Kropp. Wer sich eingehender mit der Thematik beschäftigt habe, wisse, dass es sich bei dieser Darstellung nicht um eine Übertreibung handele. Die Rhetorik der Partizipation scheine in gewissen Fällen eine willkommene Möglichkeit zur oberflächlichen Besänftigung zu sein, ohne sich auf die unbequemen Nebenfolgen (Machtabgabe/Autoritätsverlust) einer formellen Partizipation einlassen zu müssen. In München seien am Ende der Veranstaltung Fragebögen verteilt worden, auf denen Klimaanpassungsmaßnahmen, die die Wissenschaftler empfehlen würden, notiert waren und die nun von den Teilnehmenden in ihrer Akzeptabilität ausgewertet werden konnten.

Eine klassische Arbeit aus dem Jahr 1969, die zum ersten Mal auf qualitative Stufen der Partizipation aufmerksam gemacht hat, stammt von Sherry Arnstein. Arnsteins „Ladder“ versinnbildlicht die graduellen Abstufungen partizipatorischer Praktiken. Von der untersten Stufe - also qualitativ „,minderwertiger“ Partizipation - bis hin zur obersten Stufe, der qualitativ höchsten Form von Partizipation, kann man bestimmte Verfahren auf Arnsteins Ladder verorten. Auf dieser Partizipationsleiter würde das Vorgehen in München wohl am ehesten auf den unteren Stufen unter „,consultation“ oder „informing" firmieren, aber gewiss nicht unter ,citizen power" (Arnstein 1969, S. 2). Obwohl keiner der Tagungsteilnehmer in Müncheberg explizit auf Arnstein als theoretischen Hintergrund verwies, wurde das dargestellte Setting nicht naiv als Partizipation hingenommen. Stattdessen wurden die qualitativen und strukturellen Missstände des Münchener Workshops konstruktiv kritisiert.

Es bleibt fragwürdig, ob man wie Kropp die Diagnose einer misslungenen Partizipation als ein allgemeines „Strukturproblem des expertokratischen Staates" deuten kann. Schlussendlich bliebe man aber selbst bei einer erfolgreichen Begründung dieser Verallgemeinerung noch das Argument schuldig, warum und wie weit man die Wissenschaft der Öffentlichkeit annähern soll. Man kann die Auffassung vertreten, dass die Wissenschaft durch diese Einebnung ihr Spezifikum verliert, für das sie gerade geschätzt wird: ihre Autorität, die ihr durch das Einhalten nicht beliebiger methodisch-rationaler Kriterien zukommt. Dem amerikanischen Organisationssoziologen Charles Perrow (Perrow 1992, S.370) folgend, unterscheidet sich die Wissenschaft gerade in dieser Hinsicht von der ,,beschränkten Rationalität" des Laientums.

\section{Fallbeispiele}

Eine empirische Untersuchung von Anne Bundschuh und Andrea Knierim (Zentrum für Agrarlandschaftsforschung, Müncheberg) setzte an dieser Stelle an. Sie beschäftigten sich mit der Partizipation von Landwirten im Projekt „Innovationsnetzwerk Klimaanpassung Brandenburg Berlin“ (INKA BB). Das Ziel des Projekts sei es, die Trennung von Wissensproduktion und Anwendung zu überwinden. Von Seiten der Forscher erhoffe man sich $u$. a. einen Zuwachs tradierten und regionalen Wissens, sowie einen effektiven Transfer der Ergebnisse in der Anwendung - also auch Akzeptanz für die vorgeschlagenen Maßnahmen. Die transdisziplinäre Perspektive dieses Projekts fuße auf einer Kritik an den Möglichkeiten einer objektiven und wertfreien Wissenschaft.

Das Projekt INKA BB offenbarte einen erstaunlichen empirischen Befund im Hinblick auf die Demokratisierung der Wissensproduktion. Dass bei INKA BB bereits bestehende Netzwerke eine gute Grundlage für die Auswahl der Akteure, sowie einen entscheidenden Einfluss auf Motivation der beteiligten Landwirte hatten, darf als problematisch gelten. Größere landwirtschaftliche 
Betriebe, die die zeitlichen und finanziellen Ressourcen aufbringen, um sich in Netzwerken und Verbänden zu organisieren, konnten schneller und besser erreicht werden und hatten ein größeres Interesse, am Innovationsnetzwerk teilzunehmen. Dieses Ergebnis muss durch weitere Studien geprüft werden und sollte nicht unterschätzt werden. Ist es gewünscht, wie es häufig in politischen Kontexten der Fall ist, eine möglichst breite und repräsentative Akteursgruppe einzubeziehen, stößt dies, wie man sieht, offensichtlich nicht selten auf praktische Probleme. Ungleichheiten bei den Landwirten, was ihren Einfluss auf politische Entscheidungen angeht, würden durch Partizipation eher verschärft als vermindert.

Simone Hafner (Kompetenzzentrum Klimaschutz und Klimaanpassung, Universität Kassel) widmete sich in ihrem Vortrag legislativen Prozessen. Sie monierte das Vorgehen politischer Akteure in Verwaltungsverfahren. Sie beschrieb anhand einiger Beispiele (u. a. Stuttgart 21) Planungs- und Infrastrukturprojekte, in denen die Öffentlichkeit erst nach einer konkreten, bereits getroffenen Entscheidung oder unter erheblichem Druck zur Beteiligung aufgerufen wurde. Ein solches Vorgehen diene lediglich dazu, Widerspruch möglichst beiseite zu räumen und unbeschadet - ohne an den Entscheidungen etwas ändern zu müssen - an der Öffentlichkeitsbeteiligung vorbeizukommen. Ihre Empfehlungen, Konflikte frühzeitig zu erkennen, die Öffentlichkeit möglichst zeitnah in den Entscheidungsfindungsprozess einzubinden und dabei höchste Transparenz walten zu lassen, wurden einhellig aufgenommen. Es ist allerdings zu befürchten, dass das frühzeitige Erkennen von Konflikten vor politischen Entscheidungen auf nicht unerhebliche Probleme stoßen wird. Bei jeder politischen Entscheidung pro forma Partizipation anzuwenden, scheint gleichsam nicht wünschenswert zu sein.

\section{Ausblick}

Es bleibt weiterhin fraglich, inwieweit es gelingt, den genuin politischen Begriff der Partizipation und die damit verknüpften Erwartungen auf den Kontext von Forschungsprozessen zu beziehen. Die Anwendung politischer Begriffe auf die Wissenschaft spricht jedenfalls für Peter Weingarts
Diktum der Politisierung der Forschung (Weingart 1983). Grundsätzlich muss beachtetet werden, dass die dargestellten Ansätze sich allesamt auf die stark anwendungsbezogene Klimafolgenforschung beziehen. Diese decken aber keineswegs das gesamte Spektrum wissenschaftlicher Disziplinen ab. Daran anknüpfend lassen sich für weitere Untersuchungen einige Hypothesen bilden. Es könnte sein, dass sich in der Grundlagenforschung mangelnde Motive zur Partizipation auffinden lassen. Es könnte sich außerdem eine ausgeprägte autoritative Asymmetrie zwischen den Akteuren zeigen. Im Hinblick auf die Erfolgsbedingungen legislativer Prozesse, stellt sich die Frage, ob die Kriterien, die Hafner vorschlägt, zusammengenommen auch als hinreichende Prämissen für eine erfolgreiche Partizipationspraxis im Kontext der Wissenschaft gelten können. Dies sind exemplarische Fragen aus dem dargestellten Problemkreis, die mittelfristig näher untersucht werden sollten.

\section{Literatur}

Arnstein, S.R., 1969: A Ladder of Citizen Participation. In: Journal of the American Institute of Planners 35/4 (1969), S. 216-224

Perrow, Ch., 1992: Normale Katastrophen. Die unvermeidlichen Risiken der Großtechnik. Frankfurt a. M.

Weingart, P., 1983: Verwissenschaftlichung der Gesellschaft - Politisierung der Wissenschaft. In: Zeitschrift für Soziologie 12/3 (1983), S. 225-241

$\ll »$

\section{Die Energiewende in Deutschland - nicht nur eine technische Herausforderung} Rückblick auf einen Workshop im Rahmen der Helmholtz-Allianz ENERGY-TRANS

Karlsruhe, 5.-6. Juli 2012

\section{von Andreas Lösch und Jens Schippl, ITAS}

„Transition Research for the German Energy Transition" lautete das Thema eines internationalen und interdisziplinären Workshops, der im Juli 